\title{
Special Functions and Middle Convolution
}

\author{
M. Nobert Hounkonnou ${ }^{1}$, Kassim, A.M ${ }^{2}$ \\ ${ }^{1}$ University of Abomey-Calavi Republic of Benin \\ ${ }^{2}$ Lagos State University, Ojo, Lagos State, Nigeria.
}

\begin{abstract}
This article is a survey on recent studies on middle convolution for linear and nonlinear special functions [J. Phys. A: Math. Theor. 43 (2010), 175204; J. Phys. A: Math. Theor. 42 (2009), 175208; Kumamoto J. Math. 19(2006), 15-23]. Part of this survey is also based on the joint work with Y. Haraoka [J. Lond. Math. Soc. 76 (2) (2007), 438-450]. After recalling some basic facts concerning linear and nonlinear special functions, we give an overview of the recent results and current studies on middle convolution for special functions.

Keywords: Linear and nonlinear special functions; middle convolution MSC (2010): 34M55.
\end{abstract}

The main objective of this paper is to survey recent results on middle con- volution based on the papers [9, 10 , $11,15]$ and discuss computational aspects. The paper is organized as follows. In the first section we overview linear special functions such as the Gauss hypergeometric functions and the Heun functions. Next we survey nonlinear special functions, such as the Painlevé transcendents. Next we explain the notion of middle convolution. Finally, we show how to use middle convolution to derive transformations for linear and nonlinear special functions. We also explain the notion of Schlesinger systems and study how they are changed under middle convolution.

\section{Special functions}

There is no strict mathematical definition of the notion special functions". Wikipedia ${ }^{1}$ defines special functions as "particular mathematical functions which have more or less established names and notations due to their importance in mathematical analysis, functional analysis, physics, or other applications". One can also find a lot of information about special functions at Digital Library of Mathematical Functions at http://dlmf.nist.gov/, which is a recent update of Abramovich-Stegun's book"Handbook of Mathematical Functions".

\subsection{Linear special functions}

By linear special functions we understand functions defined by linear ordinary differential equations (ODEs) which have many applications in analysis, number theory, mathematical physics and other fields. One of the most important examples is the Gauss hyperheometric function. It is a solution of the hypergeometric equation given by

$$
\frac{d^{2} y(z)}{d z^{2}}+\left(\frac{c}{z}+\frac{a+b-c+1}{z-1}\right) \frac{d y(z)}{d z}+\frac{a b}{z(z-1)} y(z)=0,
$$

where $a, b, c$ are complex parameters and $y(z)$ is a complex-valued function of the independent complex variable $z$. The coefficients of the linear equation are singular at $z=0,1, \infty$. Hence, singular points of solutions (since equation is linear) are also $z=0,1, \infty$. One can check that the Gauss hypergeometric series defined by

$$
{ }_{2} F_{1}(a, b, c)(z)=\sum_{n=0}^{\infty} \frac{(a)_{n}(b)_{n}}{(c)_{n} n !} z^{n},
$$

where $(a)_{n}=a(a+1) \ldots(a+n-1), n>0,(a)_{0}=1$, is a solution of the hypergeometric equation. There exists an integral representation of the solutions of the hypergeometric equation (see, for instance, [17]) which allows calculating a monodromy group. Later on we shall explain the notion of the monodromy group as a linear representation of the fundamental group of $\mathbb{C P}^{1}-$ singular points summarizing all analytic continuations of multi-valued solutions of the equation along closed loops. There is a vast bibliography on the hypergeometric functions 
Another important example of the linear special functions is the Heun tunction [25]. It is a solution of the following equation

$$
\frac{d^{2} y(x)}{d x^{2}}+\left(\frac{c}{x}+\frac{d}{x-1}+\frac{a+b-c-d+1}{x-t}\right) \frac{d y(x)}{d x}+\frac{a b x-q}{x(x-1)(x-t)} y(x)=0 .
$$

The equation has four singularities in the complex plane at $x=0,1, t, \infty$. The parameter $q$ is called an accessory parameter (in contrast to the hypergeometric equation, it cannot be determined if the monodromy data are given). There are many open questions concerning the Heun equation (e.g., the monodromy group, integral representation of its solutions).

Remark. It is interesting to note that there are transformations between the Hcun and hypergeometric functions. A classification of at least 1 free parameter transformations between the hypergeometric function ${ }_{2} F_{1}(a, b, c)(z)$ and the Heun function $\operatorname{Hn}(t, q ; a, b, c, d)(x)$ is studied in [30]. For instance,

$$
\begin{gathered}
\operatorname{Hn}\left(9, q_{1} ; 3 a, 2 a+b, a+b+\frac{1}{3}, 2 a-2 b+1\right)(x)= \\
(1-x)^{-2 a}{ }_{2} F_{1}\left(a, b, a+b+\frac{1}{3}\right)\left(z_{1}\right), \\
\operatorname{Hn}\left(\frac{8}{9}, q_{2} ; 3 a, 2 a+b, 2 a+2 b-\frac{1}{3}, a+b+\frac{1}{3}\right)(x)= \\
=\left(1-\frac{9 x}{8}\right)^{-2 a}{ }_{2} F_{1}\left(a, b, a+b+\frac{1}{3}\right)\left(z_{2}\right), \\
18 a^{2}-9 a b+6 a, q_{2}=4 a^{2}+4 a b-2 a / 3, \\
z_{1}=-\frac{x(x-9)^{2}}{27(x-1)^{2}}, \quad z_{2}=\frac{27 x^{2}(x-1)}{(8-9 x)^{2}} .
\end{gathered}
$$

where $q_{1}=18 a^{2}-9 a b+6 a, q_{2}=4 a^{2}+4 a b-2 a / 3$,

In general, there are about 50 such transformations. Functions $z_{j}$ are Belyi functions (branched over 3 points). Among those transformations, 38 transformations are related to the invariants of elliptic surfaces with 4 singular fibers. Transformations between hypergeometric functions were classified in [29].

\subsection{Nonlinear special functions}

One of the simplest nonlinear differential equations is the Riccati equation given by $y^{\prime}=a(z) y^{2}+b(z) y+c(z)$. By a simple transformation it can be linearized. Hence, solutions are meromorphic functions (have poles in $\mathbb{C}$ ). The elliptic function $\wp(z)$ is the solution of the first order second degree nonlinear equation $y^{\prime 2}=4 y^{3}-g_{2} y-g_{3}$. The function $\wp(z)$ also has poles in $\mathbb{C}$. Note that the equation is non-linearizable. Moreover, there are a lot of applications of the function $\wp(z)$ in modern mathematics, and, hence, it can be called special. form

More generally, Painlevé (1888) proved that for the first order ODEs of the

$$
P\left(\frac{d y}{d z}, y, z\right)=0
$$

where $P$ is a polynomial in $d y / d z$ and $y$ with analytic in $z$ coefficients, the movable singularities (which depend on initial conditions) of the solutions are poles and/or algebraic branch points. It is nowadays customary to use the notions of the Painlevé and quasi-Painlevé property. Roughly, a differential equation possesses the Painlevé property if its solutions have only movable poles in $\mathbb{C}$ and quasi-Painlevé property if they have algebraic branch poirts.

In general, second and higher order nonlinear equations have more complicated movable singularities. Therefore, in order to find some more nonlinear special functions with a "good" singularity structure, one needs to understand which equations of the type

$$
\frac{d^{2} y}{d z^{2}}=R\left(\frac{d y}{d z}, y, z\right)
$$

where $R$ is a rational function of $d y / d z$ and $y$ and an analytic function of $z$, have the Painlevé property, or in other words, solutions have no movable critical points. A lot of papers by Painlevé, Picard, Fuchs, Gambier, Bureau were dedicated to the solution of the problem stated above. There exists a 
classification of such equations. There are 50 types of equations, solutions of which have only movable poles. Among them, 44 equations are integrable in terms of linear equations and elliptic functions or reducible to other six equations which are now known as the Painlevé equations:

$$
\begin{gathered}
y^{\prime \prime}=6 y^{2}+z, \quad\left(P_{I}\right) \\
y^{\prime \prime}=2 y^{3}+z y+\alpha, \quad \alpha \in \mathbb{C} \quad\left(P_{I I}\right) \\
\cdots \\
\frac{d^{2} y}{d t^{2}}=\frac{1}{2}\left(\frac{1}{y}+\frac{1}{y-1}+\frac{1}{y-t}\right)\left(\frac{d y}{d t}\right)^{2}-\left(\frac{1}{t}+\frac{1}{t-1}+\frac{1}{y-t}\right) \frac{d y}{d t}+ \\
+\frac{y(y-1)(y-t)}{t^{2}(t-1)^{2}}\left(\alpha+\beta \frac{t}{y^{2}}+\gamma \frac{t-1}{(y-1)^{2}}+\delta \frac{t(t-1)}{(y-t)^{2}}\right), \quad\left(P_{V I}\right)
\end{gathered}
$$

$\alpha, \beta, \gamma, \delta$ being arbitrary parameters. For the general background of the Painlevé equations, we refer the reader to survey papers and books $[3,12,22,28]$.

In order to find the Painlevé equations, one first needs to do tedious calculations to find a set of necessary conditions of the absence of movable critical points in solutions of a given class of equations. Next one needs to prove that those conditions are sufficient. This is a difficult problem, settled only recently by Laine, Shimomura, Steinmetz and others in the 1990th (also earlier by Hukuhara in unpublished notes). So, it took almost 100 years to rigorously prove that the solutions of the second order nonlinear ODEs $\left(P_{I}\right)-\left(P_{V I}\right)$ are actually meromorphic functions, i.e., possess the Painlevé property. The analysis of higher order equations becomes even more complicated as there are natural barriers for the analytic continuation of the solutions (e.g., the Chazy equation), and, so, there are many open problems.

The solutions of the six Painlevé equations $\left(P_{I}\right)-\left(P_{V I}\right)$ are called the Painlevé transcendents and can be regarded as nonlinear special functions (see $[2,17]$ and the references therein). They appear in many areas of modern mathematics such as mathematical physics, random matrices, enumerative algebraic geometry, Frobenius manifolds, in reductions of integrable PDEs, and others. It is interesting to note that the linear special functions and elliptic functions appear in the study of the Painlevé transcendents. For example, special solutions of the Painleve equations for special values of the parameters can be expressed in terms of the linear special functions such as (confluent) hypergeometric functions, Bessel and Airy functions. Since equation $\left(P_{V I}\right)$ has special solutions expressed in terms of the hypergeometric functions, it can be regarded as a nonlinear analogue of the hypergeometric equation. There exist nice determinant representations of solutions of the Painleve equations. The asymptotics of the Painleve equations can be expressed with the help of elliptic functions [13].

Painlevé transcendents $\left(P_{I I}\right)-\left(P_{V I}\right)$ possess Bäcklund transformations which are nonlinear recurrence relations mapping solutions of a given Painlevé equation 
parameters. Such transformations of a given equation form a group which is isomorphic to a certain affine Weyl group as shown by Okamoto [23]. Moreover, $\left(P_{I}\right)-\left(P_{V I}\right)$ admit a Hamiltonian formulation and can be written in a bilinear form. Using the differential Galois theory, Umemura recently showed that the Painlevé transcendents are irreducible to classical special functions.

The Painlevé equations (and their multivariable generalizations such as the Garnier and Schlesinger systems described below) can be expressed as the compatibility condition $Y_{x t_{j}}=Y_{t_{j} x}$, where the subscript denotes the partial derivative, of two linear systems of equations

$$
Y_{x}=A Y, \quad Y_{t_{j}}=B Y .
$$

One can find the method of isomonodromy deformations and its applications to asymptotics and the derivation of connection formulae in more details in $[13,17]$.

\section{Middle convolution for Fuchsian systems}

\subsection{Monodromy of the Fuchsian systems}

A linear system is called a Fuchsian system if it is of the form

$$
\frac{d Y}{d x}=\sum_{i=1}^{p+1} \frac{A_{i}}{x-t_{i}} Y, A_{k} \in \mathbb{C}^{n \times n} .
$$

Here we denote a $n \times n$ complex matrix by $\mathbb{C}^{n \times n}$. The poles $t_{1}, t_{2}, \ldots, t_{p+1}$ of the Fuchsian system are distinct points in $\mathbb{C P}^{1}$ (we can regard the point at infinity as a regular point here). In general, if the matrix $A_{\infty}=-\sum_{i} A_{i}$ is non-zero, the point at infinity is a singularity as well.

Reducibility in Fuchsian systems means that the matrices can be written in a "block-triangular" form

$$
\left(\begin{array}{cc}
* & * \\
0 & *
\end{array}\right)
$$

and so, there are invariant subspaces.

The set $X=\mathbb{C P}^{1} \backslash\left\{t_{1}, t_{2}, \ldots, t_{p+1}\right\}$ is open and connected. Let us fix a point $x_{0} \in X$. The fundamental group $\pi_{1}\left(X, x_{0}\right)$ is generated by the loops $\pi_{1}\left(X, x_{0}\right)=\left\langle\gamma_{1}, \gamma_{2}, \ldots, \gamma_{p+1} \mid \gamma_{1} \gamma_{2} \cdots \gamma_{p+1}=1\right\rangle$. The analytic continuation along all possible loops in the fundamental group of the (generally multivalued) fundamental solution $Y$ of the linear system is given by

$$
\gamma_{*} Y=Y M, \quad M \in G L(n, \mathbb{C}), \quad \gamma \in \pi_{1}\left(X, x_{0}\right) .
$$

The matrix $M$ is called a monodromy matrix.

Linear differential equations defined on $X$ in the complex plane have a monodromy group, which, more precisely, is a linear representation of the fundamental group of $X$, summarizing all analytic continuations along closed loops 
within $X$ :

$$
\begin{gathered}
\pi_{1}\left(X, x_{0}\right) \rightarrow \mathrm{GL}(n, \mathbb{C}), \\
\left(M_{1}, M_{2}, \ldots, M_{p+1}\right) \in(\mathrm{GL}(n, \mathbb{C}))^{p+1}: M_{1} M_{2} \cdots M_{p+1}=I_{n} .
\end{gathered}
$$

Here $I_{n}$ denotes the $n \times n$ identity matrix.

There exists a direct monodromy problem (to determine a monodromy group of a Fuchsian system) and the inverse problem (to construct a system with given regular singularities and with a given monodromy representation), which is called the Riemann-Hilbert problem $[13,17]$.

\subsection{Monodromy preserving deformations and Schlesinger systems}

Let

$$
\frac{d Y}{d x}=\left(\sum_{j=1}^{p} \frac{A_{j}}{x-t_{j}}\right) Y .
$$

The matrices $A_{k} \in G L(n, \mathbb{C})$ and $A_{\infty}:=-\left(A_{1}+A_{2}+\cdots+A_{p}\right)$ generally depend on $t_{i}$.

The isomonodromy (or, equivalently, monodromy preserving) condition means that the monodromy matrices $M_{k}$ do not depend on the positions of the poles, i.e.,

$$
\frac{d M_{k}}{d t_{i}}=0
$$

Under certain non-resonance assumptions on the eigenvalues $\theta_{k}$ of the matrices $A_{k}$ and $A_{\infty}$ one can show that the function $Y$ satisfies the following system

$$
\frac{\partial Y}{\partial t_{i}}=-\frac{A_{i}}{x-t_{i}} Y, \quad i=1, \ldots, p,
$$

in the case of the monodromy preserving deformations. The compatibility conditions of (3) and (4) are known as the Schlesinger system, or deformation equations,

$$
\begin{cases}\frac{\partial A_{i}}{\partial t_{i}}=-\sum_{k \neq i} \frac{\left[A_{i}, A_{k}\right]}{t_{i}-t_{k}}, & \\ \frac{\partial A_{j}}{\partial t_{i}}=\frac{\left[A_{i}, A_{j}\right]}{t_{i}-t_{j}}, & (i \neq j) .\end{cases}
$$

When the Fuchsian system (3) has $p=4, n=2$ and singularities $(0,1, t, \infty)$, then the Schlesinger system gives the sixth Painlevé equation $\left(P_{V I}\right)$. When $n=2$ but $p>4$, we have the Garnier systems and when $p>4$ and $n>2$, we have the Schlesinger systems. Solutions of the Garnier and Schlesinger systems can be considered as multivariable nonlinear special functions. More information can be found in [17]. 


\subsection{Rigidity}

A monodromy representation $\left(M_{1}, M_{2}, \ldots, M_{p+1}\right)$ with $M_{1} M_{2} \cdots M_{p+1}=I_{n}$ is said to be rigid, if, for any tuple $\left(N_{1}, N_{2}, \ldots, N_{p+1}\right)$ of matrices in $\mathrm{GL}(n, \mathbb{C})$ satisfying $N_{1} N_{2} \cdots N_{p+1}=I_{n}$ and $N_{j}=D_{j} M_{j} D_{j}^{-1}(1 \leq j \leq p+1)$, there exists $D \in \mathrm{GL}(n, \mathbb{C})$ such that simultaneously $N_{j}=D M_{j} D^{-1}$ for $1 \leq j \leq p+1$. In case $\infty$ is a singularity of a Fuchsian system we take $M_{p+1}=M_{\infty}$.

Example. For a Fuchsian system studied in [32] given by

$$
\left(x I_{2}-\left(\begin{array}{ll}
0 & 0 \\
0 & 1
\end{array}\right)\right) \frac{d Y}{d x}=\left(\begin{array}{cc}
\lambda & 1 \\
-\left(\lambda-\rho_{1}\right)\left(\lambda-\rho_{2}\right) & \rho_{1}+\rho_{2}-\lambda
\end{array}\right) Y
$$

the monodromy group is generated by

$$
\begin{gathered}
M_{1}=\left(\begin{array}{cc}
e(\lambda) & \nu \\
0 & 1
\end{array}\right), \\
M_{2}=\left(\begin{array}{cc}
1 & 0 \\
-\left(e(\lambda)-e\left(\rho_{1}\right)\right)\left(e(\lambda)-e\left(\rho_{2}\right)\right) e(\lambda)^{-1} \nu^{-1} & e\left(\rho_{1}+\rho_{2}-\lambda\right)
\end{array}\right),
\end{gathered}
$$

where $\nu=e(\lambda)-e\left(\rho_{1}\right)$ and $e(\varphi)=\exp (2 \pi \sqrt{-1} \varphi)$.

If $\rho_{1}=-a, \rho_{2}=-b$ and $\lambda=1-c$, and we simultaneously conjugate the matrices by a matrix from $G L(2, \mathbb{C})$, then we have a standard monodromy of the hypergeometric equation:

$$
M_{1}=\left(\begin{array}{cc}
e(-c) & e(-c)-e(-a) \\
0 & 1
\end{array}\right), \quad M_{2}=\left(\begin{array}{cc}
1 & 0 \\
e(c-b)-1 & e(c-a-b)
\end{array}\right) \text {. }
$$

Next we show that this tuple is rigid. Clearly, the matrix $M_{3}=M_{\infty}$ is defincd from the relation $M_{1} M_{2} M_{3}=I_{2}$. From the definition, if $N_{1}, N_{2}$ and $N_{3}$ are any matrices from $G L(2, \mathbb{C})$ with eigenvalues as in $M_{1}, M_{2}$ and $M_{3}$ respectively and $N_{1} N_{2} N_{3}=I_{2}$, then we can calculate explicitly the matrix $D \in G L(2, \mathbb{C})$, such that

$$
D^{-1} N_{1} D=M_{1}, \quad D^{-1} N_{2} D=M_{2} \text {. }
$$

Indeed, calculating in Mathematica ${ }^{2}$ gives

$$
D=\left(\begin{array}{cc}
-\frac{p_{1} e(2(2 a+b))(e(2 c)-1) p_{2}}{(e(2 a)-e(2 c))(e(2 c)-e(2(a+b)))} & -\frac{p_{1} e(2(a+b)) p_{2}}{e(2(a+b))-e(2 c)} \\
\frac{p_{1} e(2 a)(e(2 c)-e(2 b))}{e(2 c)-e(2(a+b))} & p_{1}
\end{array}\right)
$$

where $p_{1}$ and $p_{2}$ are arbitrary parameters.

There are several definitions of rigidity (e.g., no accessory parameters in a differential equation). However, the quickest way to check the rigidity for a given tuple of matrices is to calculate the index of rigidity and see whether it is equal to 2 or not. The index of rigidity is given by

$$
\iota=(2-(p+1)) n^{2}+\sum_{i=1}^{p+1} \operatorname{dim} Z\left(M_{j}\right)
$$


where $Z\left(M_{j}\right)$ denotes the centralizer of $M_{j}$. Recall that the centralizer of an element $z$ of a group $G$ is the set of elements of $G$ which commute with $z$.

It is known [18] that $\iota$ is even; $\iota \leq 2$ for any irreducible tuple and $\iota=2$ means rigid.

In the example above it is straightforward to see that the monodromy of the hypergeometric equation (or rank 2 Fuchsian system with 3 singularities $x=0,1, \infty)$ is rigid. Indeed, we have

$$
n=2, p=2, Z\left(M_{j}\right)=2, \iota=2 \text {. }
$$

The number $2-\iota$ can be regarded as a dimension of the moduli space of Fuchsian systems with prescribed local monodromies, i.e., a number of accessory parameters. The monodromy data determine the residue matrices in. Fuchsian systems and vice versa, up to a simultaneous conjugation by a matrix from $G L(n, \mathbb{C})$.

\subsection{Middle convolution}

By Katz' theory [18] one can obtain any irreducible rigid local system on the punctured affine line from a local system of rank one by applying a suitable sequence of middle convolutions and scalar multiplications. Katz' middle convolution functor $M C_{\mu}$ preserves important properties of local systems such as a number of singularities, the index of rigidity and irreducibility but in general changes the rank and the monodromy group. If one works with Fuchsian systems, then one needs certain additive versions of such operations on residue matrices. A trivial operation of addition is a change of the eigenvalues of the residue matrix: $A_{k} \rightarrow A_{k}+a I_{n}$, where $a \in \mathbb{C}$. Dettweiler and Reiter found an algebraic construction of Katz' middle convolution functor for Fuchsian systems and showed a relation to the Euler integral transformation $[5,6,7,8]$.

The additive version of middle convolution for Fuchsian systems depends on a scalar $\mu \in \mathbb{C}$ and is denoted by $m c_{\mu}$. It is a transformation on tuples of matrices

$$
\left.\left.\left(A_{1}, \ldots, A_{p}\right) \in\left(\mathbb{C}^{n \times n}\right)\right)^{p} \rightarrow m c_{\mu}\left(A_{1}, \ldots, A_{p}\right)=\left(\tilde{A}_{1}, \ldots, \tilde{A}_{p}\right) \in\left(\mathbb{C}^{m \times m}\right)\right)^{p}
$$

In short, the Dettweiler-Reiter construction can be summarized as follows.

(1) One starts from the original Fuchsian system

$$
\frac{d Y}{d x}=\sum_{i=1}^{p} \frac{A_{i}}{x-t_{i}} Y, A_{i} \in \mathbb{C}^{n \times n}
$$

(2) Next one performs a convolution to get the Okubo-type system

$$
\frac{d Y_{1}}{d x}=\sum_{i=1}^{p} \frac{\hat{A}_{i}}{x-t_{i}} Y_{1}, \hat{A}_{i} \in \mathbb{C}^{p n \times p n}
$$


(3) Finally, taking an irreducible part of the above system, one gets a new system

$$
\frac{d Y_{2}}{d x}=\sum_{i=1}^{p} \frac{\tilde{A}_{i}}{x-t_{i}} Y_{2}, \tilde{A}_{i} \in \mathbb{C}^{m \times m},
$$

where $m$ depends on $n, p$, and the parameter $\mu \in \mathbb{C}$. So, number of singularities of the Fuchsian system stay the same whereas matrix dimensions change.

A detailed construction of $m c_{\mu}$ is as follows. Let $\mathbf{A}=\left(A_{1}, \ldots, A_{p}\right), A_{i} \in$ $\mathbb{C}^{n \times n}$. Let us also fix points $t=t_{k} \in \mathbb{C}, k=1, \ldots, p$, and consider a Fuchsian system of rank $n$ given by (6). For $\mu \in \mathbb{C}$ one defines the convolution matrices $\hat{\mathbf{A}}=c_{\mu}(\mathbf{A})=\left(\hat{A}_{1}, \ldots, \hat{A}_{p}\right)$ by

$$
\hat{A}_{k}=\left(\begin{array}{ccccccc}
0 & \ldots & 0 & 0 & 0 & \ldots & 0 \\
\vdots & & \vdots & \vdots & \vdots & & \vdots \\
A_{1} & \ldots & A_{k-1} & A_{k}+\mu I_{n} & A_{k+1} & \ldots & A_{p} \\
\vdots & & \vdots & \vdots & \vdots & & \vdots \\
0 & \ldots & 0 & 0 & 0 & \ldots & 0
\end{array}\right) \in \mathbb{C}^{n p \times n p}
$$

such that $\hat{A}_{k}$ is zero outside the $k$-th block row. The convolution matrices define a new Fuchsian system of rank $n p$ with the same number of singularities as in the original Fuchsian system (7). This system may be reducible. In general, there are the following invariant subspaces of the column vector space $\mathbb{C}^{n p}$ :

$$
\mathcal{L}_{k}=\left(\begin{array}{c}
0 \\
\vdots \\
0 \\
\operatorname{Ker}\left(A_{k}\right) \\
0 \\
\vdots \\
0
\end{array}\right)(k \text {-th entry }), k=1, \ldots, p
$$

and

$$
\mathcal{K}=\bigcap_{k=1}^{p} \operatorname{Ker}\left(\hat{A}_{k}\right)=\operatorname{Ker}\left(\hat{A}_{1}+\ldots+\hat{A}_{p}\right) .
$$

Let us denote $\mathcal{L}=\oplus_{k=1}^{p} \mathcal{L}_{k}$ and fix an isomorphism between $\mathbb{C}^{n p} /(\mathcal{K}+\mathcal{L})$ and $\mathbb{C}^{m}$ for some $m$. The matrices $\tilde{\mathbf{A}}=m c_{\mu}(\mathbf{A}):=\left(\widetilde{A}_{1}, \ldots, \widetilde{A}_{p}\right) \in \mathbb{C}^{m \times m}$, where $\widetilde{A}_{k}$ is induced by the action of $\hat{A}_{k}$ on $\mathbb{C}^{m} \simeq \mathbb{C}^{n p} /(\mathcal{K}+\mathcal{L})$, are called the additive version of the middle convolution of $\mathbf{A}$ with the parameter $\mu$. Thus, the resulting irreducible Fuchsian system of rank $m$ is given by (8).

A relation between the convolution operation $c_{\mu}$ and the Euler integral transformation is as follows. Let $g:=\left(g_{i, j}\right)$ be a matrix with entries $g_{i, j}$ such that they are (multi-valued) holomorphic functions on $X:=\mathbb{C} \backslash T, T:=\left\{t_{1}, \ldots, t_{p}\right\} \subset$ 
$\mathbb{C}, t_{i} \neq t_{j}$ for $i \neq j$. Assume that the path $\alpha_{p+1}$ encircles an open neighborhood $U$ of $y_{0}$ and the path $\alpha_{i}$ encircles the point $t_{i}$. Then the matrix-valued function

$$
I_{\left[\alpha_{p+1}, \alpha_{i}\right]}^{\mu}(g)(y):=\int_{\left[\alpha_{p+1}, \alpha_{i}\right]} g(x)(y-x)^{\mu-1} d x, y \in U,
$$

is called the Euler transform of $g$ with respect to the Pochhamer contour $\left[\alpha_{p+1}, \alpha_{i}\right]:=\alpha_{p+1}^{-1} \alpha_{i}^{-1} \alpha_{p+1} \alpha_{i}$ and the parameter $\mu \in \mathbb{C}$.

Let $\mathbf{A}:=\left(A_{1}, \ldots, A_{p}\right), A_{i} \in \mathbb{C}^{n \times n}$ be the residue matrices of the Fuchsian system (6) and $F(x)$ be its fundamental solution. Denote

$$
G(x):=\left(\begin{array}{c}
F(x)\left(x-t_{1}\right)^{-1} \\
\vdots \\
F(x)\left(x-t_{p}\right)^{-1}
\end{array}\right)
$$

and introduce the period matrix

$$
I^{\mu}(y):=\left(I_{\left[\alpha_{p+1}, \alpha_{1}\right]}^{\mu}(G)(y), \ldots, I_{\left[\alpha_{p+1}, \alpha_{p}\right]}^{\mu}(G)(y)\right) .
$$

Then Dettweiler and Reiter showed that the columns of the period matrix $I^{\mu}(y)$ are solutions of the Fuchsian system (7) obtained by the convolution with parameter $\mu-1$, i.e., $c_{\mu-1}(\mathbf{A})$, where $y$ is contained in a small open neighborhood $U$ of $y_{0}$ (which is encircled by $\alpha_{p+1}$ ).

Katz and Dettweiler-Reiter prove that if one applies additions and middle convolutions in any order and any finite number of times to a rank one system, one gets all rigid systems with a given number of singularities.

Example. Next we show how to get the hypergeometric system from a rank one system. Let us start with

$$
\frac{d y}{d x}=\left(\frac{1+a-c}{x}+\frac{c-b-1}{x-1}\right) y .
$$

It is solved by $y(x)=x^{1+a-c}(x-1)^{c-b-1}$ up to an arbitrary parameter. If the parameter $\mu$ in middle convolution is given by $\mu=-a$, we get

$$
\frac{d}{d x}\left(\begin{array}{l}
y_{1} \\
y_{2}
\end{array}\right)=\left(\frac{\hat{A}_{1}}{x}+\frac{\hat{A}_{2}}{x-1}\right)\left(\begin{array}{l}
y_{1} \\
y_{2}
\end{array}\right)
$$

where

$$
\hat{A}_{1}=\left(\begin{array}{cc}
1-c & c-b-1 \\
0 & 0
\end{array}\right), \quad \hat{A}_{2}=\left(\begin{array}{cc}
0 & 0 \\
1+a-c & c-a-b-1
\end{array}\right) .
$$

Next, conjugating the system with

$$
S=\left(\begin{array}{cc}
c-b-1 & 0 \\
0 & 1
\end{array}\right)
$$


i.e., applying a simple transformation $Y \rightarrow S Y$, we get a new system (8) with

$$
\widetilde{A}_{1}=\left(\begin{array}{cc}
1-c & 1 \\
0 & 0
\end{array}\right), \quad \tilde{A}_{2}=\left(\begin{array}{cc}
0 & 0 \\
(1+b-c)(c-a-1) & c-a-b-1
\end{array}\right),
$$

which gives a hypergeometric equation for the first (or second) element of the vector $\left(y_{1}, y_{2}\right)^{t r}$. This system is irreducible. This means that there are no nontrivial invariant subspaces and so it is a middle convolution of the original rank 1 system (9).

\section{Recent results}

In this section we study several linear and nonlinear special functions and the corresponding Fuchsian systems. It appears that after applying middle convolution for Fuchsian systems one can get non-trivial transformations for these special functions.

\subsection{The hypergeometric equation}

The Gauss hypergeometric function is a linear special function of the isomonodromy type [20]. Indeed, the Schlesinger system (5) of the Fuchsian system

$$
\frac{d Y}{d x}=\left(\frac{A_{0}}{x}+\frac{A_{1}}{x-1}+\frac{A_{t}}{x-t}\right) Y
$$

with triangular matrices

$$
A_{k}=\left(\begin{array}{cc}
0 & 0 \\
u_{k}(t) & 0
\end{array}\right)+\theta_{k} \sigma_{3}
$$

give the following system for the functions $u_{0}, u_{1}, u_{t}$ :

$$
\frac{d u_{0}}{d t}=\frac{2 \theta_{0} u_{t}-2 \theta_{t} u_{0}}{t}, \quad \frac{d u_{1}}{d t}=\frac{2 \theta_{1} u_{t}-2 \theta_{t} u_{1}}{t-1} .
$$

Assuming additionally $u_{0}+u_{1}+u_{t}=0$, we get the Euler differential equation (or, the hypergeometric equation (1)) for $u_{0}$ with

$$
a=2 \theta_{t}, \quad b=2 \theta_{0}+2 \theta_{1}+2 \theta_{t}, \quad c=2 \theta_{0}+2 \theta_{t}+1 .
$$

One can study the effect of the application of the addition (change of the eigenvalues of the residue matrices) and middle convolution. For instance, shifting the eigenvalues of the residue matrices in (10) by addition $Y=x^{-\theta_{0}}(x-$ $1)^{-\theta_{1}}(x-t)^{\theta_{t}} Y_{1}$, we start with the system (6) with

$$
A_{1}=\left(\begin{array}{cc}
2 \theta_{0} & 0 \\
u_{0}(t) & 0
\end{array}\right), \quad A_{2}=\left(\begin{array}{cc}
2 \theta_{1} & 0 \\
u_{1}(t) & 0
\end{array}\right), \quad A_{3}=\left(\begin{array}{cc}
0 & 0 \\
u_{t}(t) & -2 \theta_{t}
\end{array}\right) .
$$

The following statement holds true [9]. 
Theorem. Let $u_{0}(t), u_{1}(t)$ and $u_{t}(t)$ with $u_{0}(t)+u_{1}(t)+u_{t}(t)=0$ satisfy the hypergeometric equations with

$$
\begin{array}{ccc}
a_{0}=2 \theta_{t}, \quad b_{0}=2\left(\theta_{0}+\theta_{1}+\theta_{t}\right), & c_{0}=2\left(\theta_{0}+\theta_{t}\right)+1 ; \\
a_{1}=2 \theta_{t}, & b_{1}=2\left(\theta_{0}+\theta_{1}+\theta_{t}\right), & c_{1}=2\left(\theta_{0}+\theta_{t}\right) ; \\
a_{t}=2 \theta_{t}+1, & b_{t}=2\left(\theta_{0}+\theta_{1}+\theta_{t}\right), & c_{t}=2\left(\theta_{0}+\theta_{t}\right)+1 .
\end{array}
$$

Then the operations of addition and middle convolution with parameter $\mu=$ $-2\left(\theta_{0}+\theta_{1}\right)$ applied to system (10) give new solutions of the hypergeometric equations

$$
\tilde{u}_{0}(t)=-\frac{2 \theta_{1} u_{0}(t)}{f(t) u_{1}(t)}, \quad \widetilde{u}_{1}(t)=\frac{2 \theta_{0}}{f(t)}, \quad \tilde{u}_{t}(t)=\frac{2 \theta_{1} u_{0}(t)-2 \theta_{0} u_{1}(t)}{f(t) u_{1}(t)}
$$

with

$$
f^{\prime}(t)=2 f(t) \frac{\theta_{1} u_{0}(t)+\left(\theta_{1}+\theta_{t}\right) u_{1}(t)}{u_{1}(t)(t-1)}
$$

and the parameters

$$
\begin{array}{rrr}
\tilde{a}_{0}=2\left(\theta_{0}+\theta_{1}+\theta_{t}\right), \quad \tilde{b}_{0}=2 \theta_{t}, & \widetilde{c}_{0}=2\left(\theta_{0}+\theta_{t}\right)+1 ; \\
\tilde{a}_{1}=2\left(\theta_{0}+\theta_{1}+\theta_{t}\right), \quad \tilde{b}_{1}=2 \theta_{t}, & \widetilde{c}_{1}=2\left(\theta_{0}+\theta_{t}\right) ; \\
\tilde{a}_{t}=2\left(\theta_{0}+\theta_{1}+\theta_{t}\right)+1, \quad \tilde{b}_{t}=2 \theta_{t}, & \widetilde{c}_{t}=2\left(\theta_{0}+\theta_{t}\right)+1 .
\end{array}
$$

More information on other cases including a connection to the Heun and Jordan-Pocchammer equations can be found in [9].

\subsection{The Heun equation}

It is convenient to differentiate the Heun equation (2) once and write down the hypergeometric system of the Heun equation

$$
\left(\begin{array}{ccc}
x & 0 & 0 \\
0 & x-1 & 0 \\
0 & 0 & x-t
\end{array}\right)\left(\begin{array}{l}
y_{1}^{\prime} \\
y_{2}^{\prime} \\
y_{3}^{\prime}
\end{array}\right)=\left(\begin{array}{ccc}
1-c & 1 & 0 \\
\alpha_{21} & -d & 1 \\
\alpha_{31} & \alpha_{32} & -\epsilon-1
\end{array}\right)\left(\begin{array}{l}
y_{1} \\
y_{2} \\
y_{3}
\end{array}\right)
$$

where

$$
\begin{aligned}
& \epsilon=a+b-c-d+1, \quad a b g=q, \\
& \alpha_{21}=(c-1) d-g_{0}, \\
& \alpha_{31}=a b(c-2)+(1-c)(d+c-2) \epsilon+(\epsilon-c+2) g_{0}, \\
& \alpha_{32}=\epsilon(d+c-2)-a b+g_{0}, \quad g_{0}=\frac{1}{t}\{\epsilon(1-c)+a b g\} .
\end{aligned}
$$

Applying middle convolution one can get the following theorem [10]. 
Theorem. The hypergeometric system of the Heun equation (11) is related via middle convolution with parameter $\mu$ and a gauge transformation to another hypergeometric system (11) with new values of the parameters given by either

$$
\begin{gathered}
\mu=a-1,\left(2-a-a_{1}\right)\left(1-a+b-a_{1}\right)=0, \\
a_{1} b_{1}=(a-2)(a-b-1), \\
c_{1}=c-a+1, d_{1}=d-a+1, \epsilon_{1}=\epsilon-a+1, \\
g_{1}=\frac{1+b-d+a(d-1+b(g-1))+(a-1)(a-c-d) t}{(a-2)(a-b-1)}
\end{gathered}
$$

or

$$
\begin{gathered}
\mu=b-1,\left(2-b-a_{1}\right)\left(1+a-b-a_{1}\right)=0, \\
a_{1} b_{1}=-(b-2)(a-b+1), \\
b_{1}=c-b+1, d_{1}=d-b+1, \epsilon_{1}=\epsilon-b+1, \\
g_{1}=-\frac{a(1+(g-1) b)+(b-1)(d-1+(b-c-d) t)}{(b-2)(a-b+1)} .
\end{gathered}
$$

It has been discussed earlier that middle convolution is related to the Euler integral transformation. Earlier studies on the Heun equation and the Euler transformation include [19]. However, the method of middle convolution gives a nice algebraic construction.

\subsection{The sixth Painlevé equation}

Although the algorithm of Dettweiler and Reiter was introduced for rigid systems, we are interested to understand what happens to general Fuchsian systems. In the case of the sixth Painlevé equation, which describes monodromy preserving deformations of rank 2 Fuchsian system with four singularities on the projective line, the algorithm yields the Okamoto birational transformation [11]. In general, as it is shown in the next section, there is an invariance of the deformation equations (or, Schlesinger system (5)) under middle convolution [15].

Let us first start with the Fuchsian system of rank 2 with 4 singularities $(n=2, p=3)$, deformation of which leads to $\left(P_{V I}\right)$ :

$$
\frac{d Y}{d x}=\left(\frac{A_{0}}{x}+\frac{A_{1}}{x-1}+\frac{A_{t}}{x-t}\right) Y
$$

with $A_{i} \in G L(n, \mathbb{C})$. In contrast to system (10) the residue matrices here are not triangular. Recall that here $t$ is a deformation parameter and we may allow it to vary by making the coefficients of the residue matrices of the system depend on $t$. The monodromy matrices do not depend on $t$ iff $y(t)$, which is a function of the coefficients of the residue matrices, satisfies $\left(P_{V I}\right)$. So, the sixth Painlevé equation govern monodromy preserving deformations of such Fuchsian systems. 
Applying middle convolution with a parameter equal to one of the eigenvalues of the matrix at infinity, we get a new system of rank $2(m=2)$. Finally, we are able to calculate [11] explicitly Okamoto's birational transformation for the solutions of $\left(P_{V I}\right)$ :

$y_{1}(t)=y-\frac{\left(\theta_{0}+\theta_{1}-\theta_{\infty}+\theta_{t}\right)(t-y)(y-1) y}{\left(\theta_{0}+\theta_{t}-1+t\left(\theta_{0}+\theta_{1}\right)\right) y-\left(\theta_{0}+\theta_{1}+\theta_{t}-1\right) y^{2}-t\left(\theta_{0}+(t-1) y^{\prime}\right)}$,

where $y=y(t)$ is a solution of $\left(P_{V I}\right)$ with

$$
\alpha=\frac{\left(\theta_{\infty}-1\right)^{2}}{2}, \beta=-\frac{\theta_{0}^{2}}{2}, \gamma=\frac{\theta_{1}^{2}}{2}, \delta=\frac{1-\theta_{t}^{2}}{2} .
$$

The parameters of the new solution $y_{1}$ are given by

$$
\begin{aligned}
& \alpha_{1}=\frac{1}{8}\left(\theta_{0}+\theta_{1}+\theta_{\infty}+\theta_{t}-2\right)^{2}, \beta_{1}=-\frac{1}{8}\left(\theta_{0}-\theta_{1}+\theta_{\infty}-\theta_{t}\right)^{2}, \\
& \gamma_{1}=\frac{1}{8}\left(-\theta_{0}+\theta_{1}+\theta_{\infty}-\theta_{t}\right)^{2}, \delta_{1}=\frac{1}{2}\left(1-\left(\theta_{0}+\theta_{1}-\theta_{\infty}-\theta_{t}\right)^{2} / 4\right),
\end{aligned}
$$

where $\theta_{i}$ are eigenvalues of the matrices $A_{i}$. It is known $[22,23]$ that the group of birational transformations forms an affine Weyl group of $D_{4}^{(1)}$ type and it is generated by 5 transformations. The meaning of 4 of them in the context of the Fuchsian system is that they are obtained by simple gauge transformations [14]. The fifth generator of the group is connected to the integral transformation, or middle convolution. Earlier studies on Okamoto's transformation by other methods include a singular manifold method [4], the Laplace transform for a system with irregular singularities [21], and others.

\subsection{Schlesinger systems}

As we have already seen in the previous subsection, the Euler integral transformation or middle convolution applied to a Fuchsian system with 4 singularities leads to the Bäcklund (or birational) transformation of the deformation equation $\left(P_{V I}\right)$ :

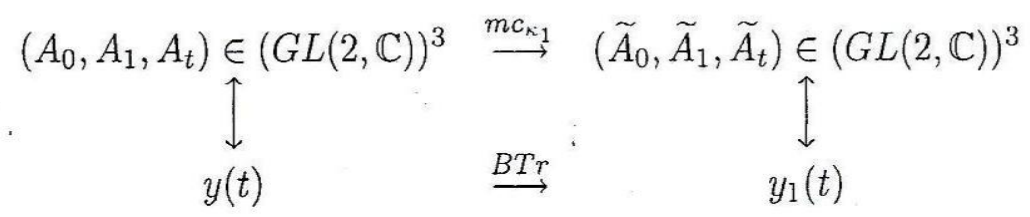

Recall that the deformation equation is the equation which should be satisfied when we require that the monodromy of the Fuchsian system is independent of the deformation parameters. It is a compatibility condition of 2 linear systems.

If we do not impose conditions on the parameter of middle convolution, we get a rank 3 system which was studied by Harnad, Mazzocco and Boalch and which proved useful in searching for new algebraic solutions of $\left(P_{V I}\right)$. We can also construct explicitly any rank Fuchsian systems deformation of which 
leads to the sixth Painlevé equation. Solutions of the Schlesinger systems can be considered as multivariable generalizations of the Painlevé equations and, hence, as new nonlinear special functions. Next we study what happens to them under middle convolution.

For a Fuchsian system (3) with $A_{p+1}=A_{\infty}=-\left(A_{1}+A_{2}+\cdots+A_{p}\right)$ one can show [15] that the isomonodromic family (solutions of the Schlesinger systems) is invariant under middle convolution.

Theorem. If for $j=1,2, \ldots, p+1$, there is no integral difference between any two distinct eigenvalues of $A_{j}$ and the Jordan canonical form of $A_{j}$ is independent of $t_{1}, t_{2}, \ldots, t_{p}$, then the systems

$$
\left\{\begin{array}{l}
\frac{\partial}{\partial t_{i}} \operatorname{tr}\left(A_{i} A_{j}\right)=-\sum_{k \neq i, j} \frac{\operatorname{tr}\left(\left[A_{i}, A_{k}\right] A_{j}\right)}{t_{i}-t_{k}} \\
\frac{\partial}{\partial t_{i}} \operatorname{tr}\left(A_{j} A_{k}\right)=\frac{\operatorname{tr}\left(\left[A_{i}, A_{j}\right] A_{k}\right)}{t_{i}-t_{j}}+\frac{\operatorname{tr}\left(A_{j}\left[A_{i}, A_{k}\right]\right)}{t_{i}-t_{k}} .
\end{array}\right.
$$

for the Fuchsian systems obtained by addition and middle convolution with parameters independent of $t_{1}, t_{2}, \ldots, t_{p}$ coincide with the system (12) for the initial Fuchsian system.

\section{Discussion}

In this paper we have discussed middle convolution for Fuchsian systems. This operation proved to be useful to study non-trivial relations between special functions $([9,10,11,15,26])$. In addition, other operations on Fuchsian systems, e.g., Yokoyama's extension [32], can be described in terms of Katz' middle convolution [24].

Recently, middle convolution for systems of linear differential equations with irregular singular points was introduced in [27] (see also [16, 31]). There are many problems for such systems: to understand the index of rigidity, to study the most interesting examples of special functions (e.g., confluent Heun and hypergeometric functions, Painlevé equations $\left.\left(P_{I}\right)-\left(P_{V}\right)\right)$ and to show the analogue of the theorem in [15] that deformation equations are invariant under this generalized middle convolution.

\section{Acknowledgements}

This paper is an extended version of the talk given at the University of Ulm at Forschungsseminar on July 21, 2010. The author is grateful to Professor Werner Balser for the invitation and hospitality during the stay.

The author is partially supported by Polish MNiSzW Grant N N201 397937. 


\section{References}

[1] G. E. Andrews, R. Askey and R. Roy, Special Functions, Encyclopedia of Mathematics and its Applications, 71. Cambridge University Press, Cambridge, 1999 .

[2] P. A. Clarkson, Painlevé equations-nonlinear special functions, J. Comput. Appl. Math. 153 (2003), 127-140.

[3] R. Conte (ed.), The Painlevé Property-One Century Later, CRM Series in Mathematical Physics, Springer, Berlin, 1999.

[4] R. Conte and M. Musette, First-degree birational transformations of the Painlevé equations and their contiguity relations, J. Phys. A 34 (2001), 10507-10522.

[5] M. Dettweiler and S. Reiter, Middle convolution and Galois realizations, in: Galois Theory and Modular Forms, vol 11, Boston, MA: Kluwer, 2004, $143-158$.

[6] M. Dettweiler and S. Reiter, An algorithm of Katz and its application to the inverse Galois problem, J. Symb. Comput. 30 (2000), 761-798.

[7] M. Dettweiler and S. Reiter, Painlevé equations and the middle convolution, Adv. Geom. 7 (2007), 317-330.

[8] M. Dettweiler and S. Reiter, Middle convolution of Fuchsian systems and the construction of rigid differential systems, J. Algebra 318 (2007), 1-24.

[9] G. Filipuk, Middle convolution and the hypergeometric equation, J. Phys. A: Math. Theor. 43 (2010), 175204.

[10] G. Filipuk, A hypergeometric system of the Heun equation and middle convolution, J. Phys. A: Math. Theor. 42 (2009), 175208.

[11] G. Filipuk, On the middle convolution and birational symmetries of the sixth Painlevé equation, Kumamoto J. Math. 19 (2006), 15-23.

[12] V.I. Gromak, I. Laine, S. Shimomura, Painlevé Differential Equations in the Complex Plane, de Gruyter Studies in Mathematics, vol. 28, Walter de Gruyter \& Co., Berlin, 2002.

[13] A. S. Fokas, A. R. Its, A. A. Kapaev and V. Yu. Novokshenov, Painlevé Transcendents. The Riemann-Hilbert Approach, Mathematical Surveys and Monographs, 128. American Mathematical Society, Providence, RI, 2006.

[14] K. Iwasaki, An area-preserving action of the modular group on cubic surfaces and the Painlevé VI equation, Comm. Math. Phys. 242 (2003), 185219 . 
[15] Y. Haraoka and G. Filipuk, Middle convolution and deformation for Fuchsian systems, J. Lond. Math. Soc. 76 ,(2) (2007), 438-450.

[16] K. Hiroe, Twisted Euler transform of differential equations with an irregular singular point, preprint arXiv:0912.5124.

[17] K. Iwasaki, H. Kimura, Sh. Shimomura and M. Yoshida, From Gauss to Painlevé. A Modern Theory of Special Functions, Aspects of Mathematics, E16. Friedr. Vieweg \& Sohn, Braunschweig, 1991.

[18] N. M. Katz, Rigid Local Systems, Annals of Mathematics Studies, 139. Princeton University Press, Princeton, NJ, 1996.

[19] A. Yu. Kazakov and S. Yu. Slavyanov, Euler integral symmetries for a deformed Heun equation and symmetries of the Painlevé VI equation, Theor. Math. Phys. 155 (2008), 252-264.

[20] A. V. Kitaev, Special functions of the isomonodromy type, Acta Appl. Math. 64 (2000), 1-32.

[21] M. Mazzocco, Irregular isomonodromic deformations for Garnier systems and Okamoto's canonical transformations, J. London Math. Soc. 70 (2004), 405-419.

[22] M. Noumi, Painlevé Equations through Symmetry, Translations of Mathematical Monographs, vol. 223, American Mathematical Society, Providence, RI, 2004.

[23] K. Okamoto, Studies on the Painlevé equations I, Ann. Mat. Pura Appl 146 (1987), 337-381; II, Japan. J. Math. 13 (1987), 47-76; III, Math. Ann. 275 (1986), 221-255; IV, Funkcial. Ekvac. 30 (1987), 305-332.

[24] 'T. Oshima, Katz' middle convolution and Yokoyama's extending operation, preprint arxiv:0812.1135.

[25] A. Ronveax (ed.), Heun's Differential Equations, Oxford University Press, Oxford, 1995.

[26] K. Takemura, Middle convolution and Heun's equation, SIGMA 5 (2009), paper 040, 22 pp.

[27] K. Takemura, Introduction to middle convolution for differential equations with irregular singularities, preprint arXiv:1002.2535.

[28] H. Umemura, Painlevé equations in the past 100 years, Amer. Math. Soc. Transl. 204 (2001), 81-110.

[29] R. Vidunas, Algebraic transformations of Gauss hypergeometric functions, Funkcial. Ekvac. 52 (2009), 139-180.

[30] R. Vidunas, R. Maier, G. Filipuk, General transformations between the Heun and Gauss hypergeometric functions, in preparation.

[31] D. Yamakawa, Middle convolution and Harnad duality, preprint arxiv:0911.3863.

[32] T. Yokoyama, Construction of systems of differential equations of Okubo normal form with rigid monodromy, Math. Nachr. 279 (2006), 327-348.

M. Nobert Hounkonnou. "Special Functions and Middle Convolution." IOSR Journal of Mathematics (IOSR-JM) 13.3 (2017): 52-67 